Géraldine Froger (dir), Tourisme durable dans les Suds collection EcoPolis, $n^{\circ}$ 11, PIE Peter Lang, 2010, 316 pages

\title{
Céline Barthon
}

\section{OpenEdition \\ Journals}

Édition électronique

URL : http://journals.openedition.org/tourisme/430

DOI : 10.4000/tourisme.430

ISSN : 2492-7503

Éditeur

Éditions touristiques européennes

\section{Édition imprimée}

Date de publication : 1 juin 2012

Pagination : 100-102

ISSN : 2109-5671

\section{Référence électronique}

Céline Barthon, «Géraldine Froger (dir), Tourisme durable dans les Suds », Mondes du Tourisme [En ligne], 5 | 2012, mis en ligne le 30 septembre 2015, consulté le 22 septembre 2020. URL : http:// journals.openedition.org/tourisme/430 ; DOI : https://doi.org/10.4000/tourisme.430

Ce document a été généré automatiquement le 22 septembre 2020.

\section{$\Theta \Theta \Theta \Theta$}

Mondes du tourisme est mis à disposition selon les termes de la licence Creative Commons Attribution - Pas d'Utilisation Commerciale - Pas de Modification 4.0 International. 


\title{
Géraldine Froger (dir), Tourisme durable dans les Suds
}

\author{
collection EcoPolis, $n^{\circ}$ 11, PIE Peter Lang, 2010, 316 pages
}

\section{Céline Barthon}

\section{RÉFÉRENCE}

Géraldine Froger (dir), Tourisme durable dans les Suds, collection EcoPolis, $\mathrm{n}^{\circ} 11$, PIE Peter Lang, 2010.

1 Dirigé par Géraldine Froger, cet ouvrage propose une série de réflexions et d'analyses critiques du modèle de développement durable appliqué au tourisme dans les pays du Sud, tout en explorant la diversité des situations et contextes géopolitiques, socioéconomiques et environnementaux des pays en développement dans lesquels le tourisme prend forme. Issu en partie d'un programme de recherche sur "la mesure des effets des formes de tourisme responsable dans différents territoires" réunissant l'Agence française de développement, le ministère des Affaires étrangères et le Cemotev (Centre d'étude sur la mondialisation, les conflits, les territoires et les vulnérabilités), les dix contributeurs (principalement économistes, mais aussi sociologue, géographe ou spécialistes des questions de développement et d'écologie politique) y analysent les contradictions et décalages entre les discours normatifs du tourisme durable et sa mise en pratique sous différentes formes dans les pays du Sud.

2 Pour autant, l'introduction n'échappe pas à l'incontournable classification des formes de tourisme reposant sur des pratiques et des valeurs considérées par principe comme durables (tourisme culturel, de nature, écotourisme, solidaire, responsable...) bien que nombreux chercheurs - y compris parmi les auteurs de l'ouvrage - aient démontré les faiblesses et limites de ces alter-tourismes dans leur capacité à faire interagir les dimensions économique, environnementale et sociale du développement durable. Notons d'ailleurs que "les risques d'un développement incontrôlé du tourisme" - sous- 
entendu, de masse - sur le développement et l'environnement existent également, à des échelles territoriales plus fines, pour ces formes dites alternatives.

Reprenant cette typologie, les études de cas présentées dans les deuxième et troisième parties font donc la part belle à l'écotourisme et au tourisme rural communautaire sur deux aires géographiques bien distinctes: l'Afrique et l'Amérique latine. Le lecteur ne sera pas étonné de retrouver parmi les terrains investigués des pays comme Madagascar (Djohary Andrianambinina) ou le Costa Rica (Marie Bonnin), hauts lieux, pour des raisons différentes, de l'écotourisme où se développent en parallèle des initiatives de tourisme rural communautaire - les deux n'étant pas incompatibles si l'on considère les aires naturelles protégées comme des espaces habités. C'est également le cas dans les régions du nord-ouest et du nord-est de la Namibie, où l'octroi aux communautés rurales de droits d'usage et d'exploitation des ressources naturelles sous la forme de conservancies a donné une légitimité spatiale et institutionnelle aux initiatives de tourisme communautaire (Renaud Lapeyre). Plus "culturels" sont les projets développés dans la Quebrada de Humahuaca, en Argentine, où le classement sur la liste du patrimoine mondial de l'Unesco a contribué à une prise de conscience locale des aménités culturelles et paysagères de cette vallée préandine à l'origine du développement d'un tourisme culturel et communautaire (Émilie Wolff). Enfin, plus proche de l'Europe, Mehdi Marzouki interroge la durabilité du tourisme sur les sites de Tozeur et de Tabarka (région ouest de la Tunisie) dont le développement, initialement conçu de manière différenciée, en opposition au tourisme balnéaire de masse, reste finalement étroitement dépendant de ce dernier.

Pour chacune de ces études, une même grille de lecture "coût-bénéfice", adaptée de Weaver (2001), est systématiquement mobilisée afin d'analyser les retombées positives et négatives du tourisme - et non du "tourisme durable" comme indiqué - en fonction de ses effets directs et indirects dans les trois champs du développement durable (économiques, socioculturels et environnementaux). Le contexte politique et territorial dans lequel ces initiatives de tourisme présupposées durables prennent place est également appréhendé, puisqu'il peut être facteur de succès comme d'échec. Aussi les cinq contributions reposant sur les études de cas reprennent-elles le même plan: présentation du contexte, évaluation des impacts et des facteurs de réussite et d'échec.

Indéniablement, l'utilisation de cette grille présente des vertus pédagogiques puisqu'elle autorise, de fait, une approche comparatiste et un instantané des relations souvent contradictoires entre "tourisme et économie", "tourisme et environnement" et "tourisme et développement social et culturel". Elle souligne aussi des différences d'appréciations de certains processus en fonction des études - et peut-être aussi des auteurs. Par exemple, la diversification des activités permise par le tourisme est à la fois interprétée comme un facteur de vulnérabilité économique supplémentaire ou de réduction de celle-ci, sans que l'on sache sur quels fondements méthodologiques ces constats ou conclusions reposent. Il est d'ailleurs regrettable que cette approche de la vulnérabilité ne soit développée que dans le seul registre des impacts économiques, alors qu'elle se décline également sur les champs environnementaux - très en retrait dans la majorité des études - et sociaux. Mais, pour cela, il aurait fallut faire interagir les trois dimensions du développement durable, ce qu'une grille de lecture les dissociant ne peut mettre en œuvre. Ainsi, comme le soulignent David DumoulinKervran et Sébastien Velut dans l'introduction de la troisième partie: "l'approche des impacts devrait s'enrichir d'une pensée des processus et des interactions dans la durée [...] dans 
une perspective à la fois historique et multi-scalaires pour mieux rendre compte des évolutions et pouvoir, le cas échéant, les orienter." C'est, à notre avis, la principale faiblesse de ces études, la durabilité du tourisme ne pouvant s'évaluer uniquement par ses impacts sur chacune des composantes du développement durable prises de manière isolée à un instant $t$.

6 Alors que les deuxième et troisième parties présentent une certaine homogénéité d'un point de vue méthodologique, la première, intitulée "diagnostic et réflexions sur le tourisme durable dans les Suds", s'apparente davantage à une juxtaposition d'articles dont le lecteur a bien du mal à identifier le fil conducteur. Les potentialités et les limites du tourisme durable y sont abordées sous des angles très divers: celui de la diversification du tourisme et du lien social qu'il peut créer, sous réserve de répondre aux enjeux économiques et écologiques des territoires et de créer des réseaux de liens sociaux pour Étienne Rodary; celui de l'empreinte écologique, utilisée ici comme un indicateur de durabilité globale du tourisme, pour Mehdi Marzouki, Jérôme Ballet et Géraldine Froger). Sans surprise, nous apprenons, à l'aide de calculs savants, que plus la distance entre pays émetteur et récepteur est grande, plus les formes de tourisme qui mettent en relation ces pays sont limitées (en termes de capacité d'accueil ou de charge) et plus l'empreinte écologique est élevée. L'écotourisme aux Seychelles dénomination que l'on pourrait longuement discuter - est donc écologiquement moins durable, d'un point de vue global, que le tourisme de masse en Tunisie. Le précepte "penser global, agir local" du développement durable trouverait ainsi ses limites, dans un contexte mondialisé du tourisme...

7 Cette approche scalaire est mobilisée indirectement par Denis Requier-Desjardins dans son analyse du tourisme rural comme élément contributeur du développement local durable dans les pays du Sud, lorsqu'il aborde les relations entre le tourisme et l'économie résidentielle et présentielle. Le tourisme, en effet, détermine des formes spécifiques de développement local en diversifiant et métissant les activités (plus exclusivement agricoles dans les espaces ruraux, y compris dans les pays du Sud), en créant des "paniers de biens" et de nouveaux "systèmes localisés d'activité" jouant sur la qualité et la qualification des territoires, d'une part, et en s'appuyant sur l'effet multiplicateur des dépenses touristiques alimentées par une base présentielle, d'autre part. D'où le rôle déterminant de l'existence d'un marché national ou régional (présent en Amérique Latine, moins en Afrique) alimenté par les classes moyennes à moyennessupérieures urbaines afin de limiter l'effet de fuite constaté lorsque la fréquentation repose uniquement sur une clientèle internationale. D'où le rôle, tout aussi important, des migrations de retour, transitoires ou permanentes, susceptibles d'accroître la valorisation des territoires ruraux. Car, au-delà des formes de tourisme qui prennent forme sur ces espaces ruraux, c'est bien leur capacité à produire du développement qu'il s'agit d'évaluer : un développement socialement et écologiquement responsable, mais aussi économiquement viable. Ainsi l'écotourisme dans les pays du Sud oscille-t-il entre mythes et réalités (Géraldine Froger et Mehdi Marzouki et Jérôme Ballet) dont les constructions et analyses mériteraient d'être renouvelées.

8 Finalement, cet ouvrage s'apparente à des actes de colloque. Riche en contributions diverses - en qualité comme en quantité -, il nous invite à poursuive la réflexion sur les modalités de développement durable du tourisme dans les pays du Sud plus que sur les formes de tourisme dit durable à proprement parler. 


\section{AUTEURS}

CÉLINE BARTHON

Université d'Angers 